\title{
Effects of COVID-19 on male sex function and its potential sexual transmission
}

\author{
Héctor Rodriguez Bustos, Gonzalo Bravo Maturana, Felipe Cortés-Chau, Joelle Defaur Torres, \\ Felipe Cortés-Pino, Pablo Aguirre, Camilo Arriaza Onel
}

Laboratorio de Morfología, ICBM, Facultad de Medicina, Universidad de Chile, Santiago, Chile.

\begin{abstract}
Summary Introduction: Severe Acute Respiratory Syndrome Coronavirus 2, (SARS-CoV-2) was first identified by the Chinese Centers for Disease Control and Prevention on January 8, 2020 and was declared as a global pandemic on March 11, 2020 by WHO. SARS-CoV-2 uses the Angiotensin-converting enzyme 2 (ACE2) receptor as an entry route, associated with the transmembrane serine protease protein (TMPRSS2), which makes the testis and particularly spermatogenesis potentially vulnerable, since this tissue has high expression of ACE2.

Material and methods: We performed a systematic literature review by electronic bibliographic databases in Pubmed, Scopus and ScienceDirect up to August 2020 about the effect of SARS-CoV-2 on male sexual function and its transmission, to assess possible repercussions on sex organs and the existence of a sexual transmission path.

Results: Although SARS-CoV-2 presence has not been found in testicle samples, it has been demonstrated that it causes histological changes compatible with orchitis, and sex hormone disturbances. TMPRSS2 is up-regulated in prostate cancer where it supports tumor progression, thus these patients may have a higher risk of SARS-CoV-2 infection. TMPRSS2 inhibitors may be useful for the treatment or prevention of COVID-19. No viral material has been found in blood or semen, however it has been proven to be present in stool and saliva. Conclusion: The male reproductive system would be highly vulnerable and susceptible to infection by SARS-CoV-2 given the expression of the ACE2 receptor in somatic and germ cells. The seminal fluid would remain free of viral presence in patients with COVID-19. Regardless, non-genital sex could be an important source of viral transmission. In assisted reproduction techniques all necessary tests must be carried out to ensure the donor is free of the virus at the time of collection and handling of the seminal sample.
\end{abstract}

KEY WORDS: COVID-19; Andrology; Fertility; Testis; Semen; Human.

Submitted 22 August 2020; Accepted 5 October 2020

\section{INTRODUCTION}

On January 8, 2020, a new coronavirus was identified at the Chinese Centers for Disease Control and Prevention. It was named as Severe Acute Respiratory Syndrome Coronavirus 2, abbreviated as SARS-CoV-2. The disease that is caused by this virus was denominated Coronavirus Disease 2019 (COVID-19) and declared as notifiable by
WHO (1). Once COVID-19 is installed, its severity and prognosis depend on multiple factors, including sex (male), age (over 60 years), and the presence of pre-existing diseases (diabetes, hypertension, and cardiovascular diseases) (2).

Coronaviruses ( $\mathrm{CoV}$ ) belong to the coronaviridae family and are characterized by being positive single-stranded RNA type, which encodes for topographically positioned proteins: spicules (S), envelope (E), membrane (M), and nucleocapsid (N). In pathogenicity, $\mathrm{S}$ proteins are essential to enter the host cell (3).

The biology of the testicle represents an ideal model for the study and evaluation of pathologies, given that events of mitosis, meiosis, and cell differentiation occurring in it, in addition to its endocrine regulation. Also, the testicle is highly sensitive to variations in body homeostasis. Particularly in the case of SARS-CoV-2, which uses the Angiotensin-converting enzyme 2 (ACE2) receptor as an entry route, associated with the transmembrane serine protease protein (TMPRSS2), makes the testis and particularly spermatogenesis potentially vulnerable, since this tissue has high expression of ACE2 receptor $(4,5)$.

\section{Methods}

Review of studies and international experience

A systematic review of the literature was performed using the electronic databases Pubmed, Scopus and Science Direct to assess the effect of SARS-CoV-2 on male sexual tract and function, and its possible sexual transmission. Multiple permutations of the following keywords were used: "COVID-19, SARS-CoV-2, transmission, testis, sperm, spermatogenesis, fertility cryopreservation, hormones, testosterone, sexual health". The search was focused in original articles published between December 2019 and August 2020. There were also included papers that were found relevant to the research team in previous research. Editorials, correspondence, case reports were excluded. The experience in reproductive and testicular biology, spermatogenesis and seminal analysis of the work team was also considered in the making of this narrative review.

\section{Results}

Across all databases a total of 371 articles were found. After reviewing the title and abstract of these, 46 papers 
were preliminary selected. The selected papers were critically read, 20 were found to be the most relevant for the purpose of this review.

\section{ACE2}

The ACE2 system plays an essential role in protecting cellular homeostasis against viral infection, specifically in COVID-19 infection and is widely distributed in the heart, kidney, lung, and testicles (4). ACE2 participates as an antagonist of the activation of the classic ReninAngiotensin System (RAS), protecting against cellular damage in hypertension, diabetes and some heart diseases. SARS-CoV-2 is characterized by occupying the ACE2 cell membrane receptor to enter the cell.

In the lung, SARS-CoV-2 invades pneumocytes and macrophages using ACE2 receptors (4), although ACE2 normally has a protective effect against tissue damage. ACE2 is present in non-immune cells, eg, respiratory and digestive tract epithelia, endothelial cells, cells of the simple cubic epithelium of the proximal convoluted tubules of the kidney, and brain neurons, as well as in immune cells such as monocytes and macrophages (3). In other cell types the virus enters its host, but with lower affinity.

The expression of ACE2 in adult human testis occurs predominantly in spermatogonial cells, Sertoli cells of the tubular compartment and Leydig cells in the interstitial compartment.

These cells are also enriched with intercellular junctions (Leydig and Sertoli cells) and have low mitochondria concentration. The above described indicates that there are cells in the testicle that can potentially be targeted for infection with the SARS-CoV-2 virus.

It has been described a high expression of ACE2 in different types of spermatogenesis cells, such as spermatogonia, early and late spermatocytes, round spermatids, and elongated spermatids $(2,4)$. It has also been described in somatic endothelial testis cells, Leydig cells, and monocytes, thus concluding that the human testis is a potential receptor for SARS-CoV-2 infection.

\section{Testicle}

In patients with the diagnosis of testicular cancer, or being treated for it, medical care should be taken as a priority in-hospital care, although in general, they are at low risk for developing a severe COVID-19 infection (6). Although SARS-CoV-2 is the cause of multiple organic alterations, Wang et al. (1) states that the male reproductive system may not be immune to alterations. Dramatic changes in sex hormones are cited, suggesting a potential impairment of gonadal function (7). In parallel, it is mentioned that in the analysis of testicular biopsies ( $\mathrm{n}=$ 6) of patients who died of SARS, several histopathological processes compatible with orchitis of variable intensity, such as apoptosis in the different types of spermatogenesis cells (cytos I and cytos II), absence or scarce sperm in the seminiferous tubules, a thickened peritubular compartment, and leukocyte infiltration, specifically citing the basal lamina (8). These findings should be compared with the normal testis morphology, as seen in Figure 1. Viral presence was not detected through in situ hybridization, but abundant $\operatorname{IgG}$ precipitates were detected (8). Finally, it is suggested that something similar could happen with COVID-19 (1).

Yang et al. (9) analyzed testicular biopsies $(\mathrm{n}=11)$ of patients who died from COVID-19. Morphological changes suggestive of damage to the seminiferous tubules were reported, such as Sertoli cell swelling, vacuolation, cytoplasmic rarefaction, and detachment from tubular basement. Interstitial changes consistent with viral orchitis were also described, with edema and mild lymphocytic inflammation, accompanied by a decrease in Leydig cell number. Although these findings would suggest SARSCoV-2 may be the causative agent of testicular tissue damage, the effect of hyperthermia, secondary infection, hypoxia, and steroidal therapy cannot be excluded. It is worth mentioning that no evidence of the presence of SARS-CoV-2 was found in the samples (9).

\section{COVID-19 effect on sex hormones}

Ma et al. (7) described that in SARS-CoV-2 infection dramatic changes are developed in sex hormones, Testosterone (T) and Luteinizing Hormone (LH), and in $\mathrm{T} / \mathrm{LH}$ ratio. In this study, sex-related hormones were compared between reproductive-aged men with SARSCoV-2 infection and age-matched healthy men (7).

It was found that COVID-19 patients had significantly

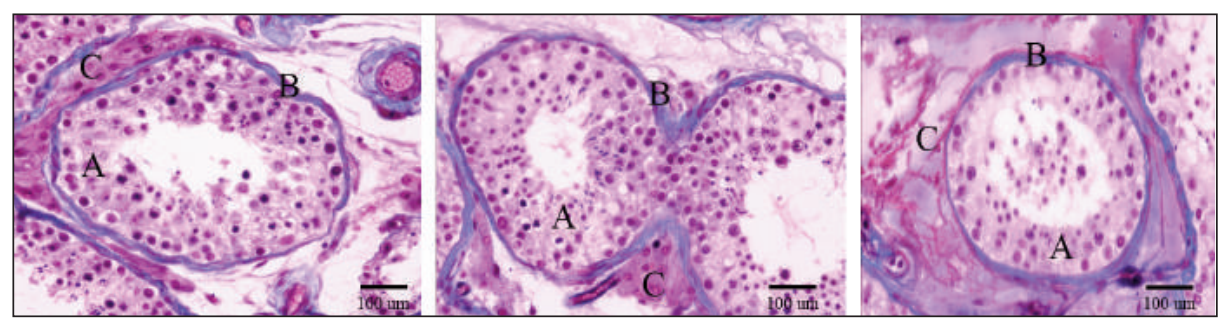

Figure 1.

Testicular biopsies. Histology of the normal human testis (young and fertile adult male). Periodic acid-Schiff (PAS), hematoxylin $(H)$, and Alcian Blue staining. A. The tubular compartment includes a simple columnar epithelium formed by Sertoli cells with a noticeable basal lamina of collagen IV, plus cytogenic epithelium forming spermatogenesis. Human spermatogenesis includes six cell stages, where 4 cycles complete a wave, including processes of mitosis (from dark spermatogonia to spermatocytes I), meiosis (with the most abundant pachytene stage) and cell differentiation (spermatocytes II, round and elongated spermatids). Therefore, there are few tubules in whose lumen sperm are found (<10\%). B. The peritubular compartment that limits the periphery of the seminiferous tubules is composed of 2-5 very thin layers of elongated cells arranged circularly. These cells have fibromuscular contractile characteristics. C. The interstitial compartment is the place where Leydig cells are located. Leydig cells are abundant and normally distributed in clumps. They have a rounded and central nucleus, and a strongly acidophilic cytoplasm. Additionally, c-kit cells, interstitial cells of Cajal and fibroblasts can be found in the interstitium. 
higher serum LH and significantly decreased T/LH and FSH/LH ratio. No statistical difference in serum T, E2 or FSH was detected between both groups (7).

Considering that $\mathrm{T}$ levels were similar between both groups, LH levels were dramatically increased in COVID-19 group, resulting in decrease in T/LH ratio. This could suggest that SARS-CoV-2 infection has an effect on the testes similar to what is seen in early stages of primary hypogonadism, in which $\mathrm{T}$ levels are maintained by the stimulated production of $\mathrm{LH}$ due to the impairment of negative feedback (7).

The findings of similar FSH, E2 and T/E2 between both groups could be interpreted as unaltered suppression of FSH by inhibin B secreted by Sertoli cells, and conserved peripheral aromatization of androgens. This suggests that SARS-CoV-2 infection has an important effect on Leydig cell function, opposed to what appears to be an unscathed Sertoli cell function (7).

\section{Prostate}

According to the epidemiological information available on COVID-19, the disease is more prevalent in male patients, with a higher incidence compared to female patients. Even more in the elderly, where prostate cancer is prevalent (2).

Montopoli et al. (10), described an action of SARS CoV-2 through the use of the ACE2 receptor and its interaction with the virus protein $\mathrm{S}$ by the transmembrane protein TMPRSS2. TMPRSS2 inhibition may work to block or decrease the severity of SARS-CoV-2 infections.

Interestingly, TMPRSS2 is an androgen-regulated gene that is up-regulated in prostate cancer where it supports tumor progression and is involved in a frequent genetic translocation with the ERG gene. Thus, patients with prostate cancer have a higher risk of SARS-CoV-2 infections compared to patients without cancer. However, prostate cancer patients receiving androgen deprivation therapy appear to be partially protected from SARS-CoV-2 infections.

Dana et al. (11) described that TMPRSS2 is the most common gene involved in primary prostate cancer, indicating that the administration of TMPRSS2 inhibitors, which are currently used for prostate cancer, may be useful for the treatment or prevention of COVID-19.

Regulators of the expression of TMPRSS2 protein in the lung may overlap with the risk factors for prostate cancer with TMPRSS2-ERG-positive fusion. Explanation of sexspecific difference in the overall incidence of COVID-19 should consider possible differences in laboratory tests for SARS-CoV-2 and the presence and activity of TMPRSS2 - ERG associated with prostate cancer, as well as strong TMPRSS2 regulation. Androgen levels could suggest that TMPRSS2 could partially explain the male prevalence in the COVID-19 pandemic. When studying mRNA levels, the constitutive expression of TMPRSS2 in lung tissue does not appear to differ between men and women. It is tempting to speculate that androgen receptor inhibitory therapies could reduce COVID-19 susceptibility to lung symptoms and mortality (12).

\section{Gastrointestinal transmission}

Additionally, Zhang et al. (13) described that ACE2 is highly expressed in enterocytes, esophageal and colonic epithelium, suggesting possible fecal-oral transmission of SARS-CoV-2. This hypothesis is sustained by the presence of genetic material of the virus in the faeces during respiratory disease and a few days after recovery (13).

Detection of SARS-CoV-2 by saliva has become an alternative option for viral load sampling for diagnosis and follow-up in COVID-19 patients, providing a safer and easier alternative for medical personnel to obtain samples, in comparison to nasopharyngeal swabbing (14).

Viral strains can be found in saliva up to 29 days after infection. Therefore COVID-19 can be transmitted by saliva directly or indirectly, even among patients without cough or other respiratory symptoms (14). Hence, since COVID-19 is present and detectable in saliva, this would indicate that it could potentially be transmitted through oral sex.

\section{Blood transmission}

Chang et al. (15), described that in SARS, in some patients, depending on the state of the evolution of the disease, it was possible to find the virus in blood plasma and lymphocytes, although in very low concentrations. Transmission of the disease through blood or plasma transfusion has not yet been described. Something very similar occurs in cases of MERS and COVID-19.

Additionally, others describe that no cases have been reported yet and there is no information on the transmission of the virus through blood transfusion therapies. However, the absence of risk of blood transfusion or its therapeutic by-products cannot be guaranteed.

It has been recommended and suggested the use of riboflavin and ultraviolet light, allowing to reduce the viral titers that could be present in whole blood to be used for transfusions. Blood products with pathogen reduction may be a safer option for critically ill patients with COVID-19, particularly those in high-risk categories $(16,17)$.

\section{Cryopreservation of spermatozoids}

In men who developed infections caused by viruses with viremic phases like Zika, Ebola and Mumps viruses, it is possible to find viral particles in semen for several months after their recovery (18). Therefore, when developing sperm cryopreservation processes, it is important to include laboratory protocols that allow ruling out any infection or presence of remains of viral particles.

It is necessary to remember that the seminal fluid incorporates the functional and secretory results of several important organs, such as the prostate and seminal glands, and the testicular fluid.

Yakass et al. (19) indicate that given the structure of the SARS-CoV-2 virus as enveloped RNA viruses, it could still be viable if it is cryopreserved and then heated to be transferred, even in cryopreservation in liquid nitrogen, similar to what is seen in Influenza virus infection. Therefore, the authors recommend always taking all necessary and sufficient safeguards.

Although the presence of active viral particles of SARS$\mathrm{CoV}-2$ has not yet been demonstrated in spermatogenesis or semen, there are warnings and procedures for sperm management obtained from donors for assisted fertilization procedures, suggesting a waiting require- 
ment of at least 2 weeks if the donor has recovered from the disease and distancing if it comes from geographic areas with high risk of infection (20).

\section{Reproductive health in COVID-19 pandemic}

In a state of health emergency such as the COVID-19 pandemic, it is necessary to identify the biomedical contributions necessary to assist the population in their reproductive health care, considering both the population in confinement and the patients recovered from the disease.

During strict confinement, behavioral adaptations occur inside the household that are generally associated with an overuse of digital technologies (telework and leisure). This implies a great overexposure to blue light (daylight and LED light from technological devices) that eventually and sooner rather than later leads to alteration in circadian rhythms, preventing the normal functioning of the pineal gland. This condition has been shown to alter the function of the ovary (21) and the testis (22), in addition to mental disorders.

Since the pandemic was declared by the WHO, the most affected countries in Europe have been Italy and Spain. Currently, both countries have received the contributions of experts in reproductive health and their association with COVID-19. In Italy the Italian Association of Andrology (23) and in Spain the Association for the study of the Biology of Reproduction and the Spanish Association of Andrology, Sexual and Reproductive Medicine (24) were attentive to the recommendations of the $\mathrm{WHO}$ and new related scientific literature.

\section{The complexity of andrology research in pandemic times}

In South America, the pandemic has been causing health havoc similar to Europe, but with a lag of around 2 months. For example, in Chile strict quarantine confinement was decreed and implemented from the second week of March to date (August). With the foregoing, only activities classified as essential have been carried out, within which university teaching or research has not been included. Most medical services have been targeted or have been converted to COVID-19 response mode. Therefore, little to no scientific research has been possible to develop in this regard. Additionally, the management of the legal aspects regarding the handling of corpses and patient samples in pandemic states means that access to biological material is restricted.

\section{Conclusions}

The male reproductive system would be highly vulnerable and susceptible to infection by SARS-CoV-2 given the expression of the ACE2 receptor, both in somatic cells (Leydig, Peritubular, and Sertoli cells), as well as in almost all germ cells in their mitotic, meiotic, and differentiation stages. Added to this is the fact that the expression of TMPRSS2 is regulated by androgens.

However, to date, no studies have been published that have found evidence of the virus in the testis, prostate tissue, or semen. Therefore, the seminal fluid would still remain free of viral presence in patients with COVID-19. Regardless, non-genital sex could be an important source of viral transmission in asymptomatic and mild symptomatic patients.

It is imperative to rule out that the sperm to be used in assisted reproduction techniques may come from donors who have suffered from COVID-19. All necessary tests must be carried out to ensure that the donor is free of the virus at the time of collection of the semen sample, during the procedures and handling of the samples.

In case of prostate cancer with indication for radical prostatectomy, if the patient has reproductive intentions, the option of using sperm cryopreservation should be proposed, after studying the absence of active seminal SARS-CoV-2.

Considering the difficulty to obtain tissue samples of COVID-19 deceased patients, due to the strict protocols and limitation put in order by governmental institutions, mostly all histological research has been done in testis, with almost no studies describing the effects of SARSCov-2 in prostate and seminal vesicles, leaving a huge gap in the body of evidence concerning this virus impact on the male reproductive tract.

\section{References}

1. Wang S, Zhou X, Zhang T, Wang Z. The need for urogenital tract monitoring in COVID-19. Nat Rev Urol. 2020; 17:314-315.

2. Cheng $H$, Wang $Y$, Wang GQ. Organ-protective effect of angiotensin-converting enzyme 2 and its effect on the prognosis of COVID-19. J Med Virol. 2020; 92:726-730.

3. Magrone T, Magrone M, Jirillo E. Focus on receptors for Coronaviruses with special reference to angiotensin-converting enzyme 2 as a potential drug target - A perspective. Endocr Metab Immune Disord Drug Targets 2020; 20:807-811.

4. Li MY, Li L, Zhang Y, Wang XS. Expression of the SARS-CoV-2 cell receptor gene ACE2 in a wide variety of human tissues. Infect Dis Poverty. 2020; 9:45.

5. Hoffmann M, Kleine-Weber H, Schroeder S, et al. SARS-CoV-2 cell entry depends on ACE2 and TMPRSS2 and is blocked by a clinically proven protease inhibitor. Cell. 2020; 181:271-280.

6. Secin FP. Priorities in testis cancer care during Covid-19 pandemic. Int Braz J Urol. 2020; 46:79-85.

7. Ma L, Xie W, Li D, et al. Effect of SARS-CoV-2 infection upon male gonadal function: A single center-based study. medRxiv. 2020.

8. Xu J, Qi L, Chi X, et al. Orchitis: A complication of severe acute respiratory syndrome (SARS). Biol Reprod. 2006; 74:410-416.

9. Yang M, Chen S, Huang B, et al. Pathological findings in the testes of COVID-19 patients: clinical implications. Eur Urol Focus. 2020; 6:1124-1129.

10. Montopoli M, Zumerle S, Vettor R, et al. Androgen-deprivation therapies for prostate cancer and risk of infection by SARS-CoV-2: a population-based study $(N=4532)$. Ann Oncol. 2020; 31:10401045.

11. Maleki Dana P, Sadoughi F, Hallajzadeh J, et al. An insight into the sex differences in COVID-19 patients: what are the possible causes? Prehosp Disaster Med. 2020; 35:438-441.

12. Stopsack KH, Mucci LA, Antonarakis ES, et al. TMPRSS2 and COVID-19: Serendipity or opportunity for intervention? Cancer Discov. 2020; 10:779-782. 
13. Zhang JC, Wang S Bin, Xue YD. Fecal specimen diagnosis 2019 novel coronavirus-infected pneumonia. J Med Virol. 2020; 92:680682.

14. Aponte Mendez M, Rivera Marval EK, Talebzade Toranji M, et al. Dental care for patients during the Covid-19 outbreak: a literature review. Int J Sci Res Dent Med Sci. 2020; 2:42-45.

15. Chang L, Yan Y, Wang L. Coronavirus Disease 2019: Coronaviruses and blood safety. Transfus Med Rev. 2020; 34:75-80.

16. Ragan I, Hartson L, Pidcoke $H$, et al. Pathogen reduction of SARS-CoV-2 virus in plasma and whole blood using riboflavin and UV light. PLoS One. 2020; 15:e0233947.

17. Keil SD, Ragan I, Yonemura S, et al. Inactivation of severe acute respiratory syndrome coronavirus 2 in plasma and platelet products using a riboflavin and ultraviolet light-based photochemical treatment. Vox Sang. 2020; 115:495-501.

18. Feldmann H. Virus in semen and the risk of sexual transmission. N Engl J Med. 2018; 378:1440-1441.

19. Yakass MB, Woodward B. COVID-19: should we continue to cryopreserve sperm during the pandemic? Reprod Biomed Online. 2020; 40:905

20. Esteves SC, Lombardo F, Garrido N, et al. SARS-CoV-2 pandemic and repercussions for male infertility patients: A proposal for the individualized provision of andrological services. Andrology. 2021; 9:10-18

21. Espino J, Macedo M, Lozano G, et al. Impact of melatonin supplementation in women with unexplained infertility undergoing fertility treatment. Antioxidants (Basel). 2019; 8:338.

22. Sun T-C, Li H-Y, Li X-Y, et al. Protective effects of melatonin on male fertility preservation and reproductive system. Cryobiology. 2020; 95:1-8.

23. Maretti C, Privitera S, Arcaniolo D, et al. COVID-19 pandemic and its implications on sexual life: Recommendations from the Italian Society of Andrology. Arch Ital Urol Androl. 2020; 92:73-77.

24. José FG, González JGÁ, Molina JMC, et al. Infección por SARSCoV-2: implicaciones para la salud sexual y reproductiva. Rev Int Androl. 2020; 18:117-23.

\section{Correspondence}

Héctor Rodriguez Bustos, MV, MSc, DBM, PhD (Corresponding Author) hector3@uchile.cl

Gonzalo Bravo Maturana, MD gonzalo.bravo.m@ug.uchile.cl

Felipe Cortés-Chau, MD

felipe.cortes.c@ug.uchile.cl

Joelle Defaur Torres, MD

joelle.defaur@ug.uchile.cl

Felipe Cortés-Pino, MD

felipe.cortes.p@ug.uchile.cl

Pablo Aguirre, MD

pablo.aguirre@ug.uchile.cl

Camilo Arriaza Onel, MD

carriaza@med.uchile.cl

Laboratorio de Morfología, ICBM, Facultad de Medicina. Universidad de Chile, Avda, Independencia 1027, Santiago (Chile) 